\title{
Successful Desensitization to Oxaliplatin After a Single Initial Dose of Omalizumab in a Patient With Elevated IgE Levels
}

Penella $\mathrm{J}^{1}$, Quan $\mathrm{P}^{1}$, Carvallo A ${ }^{1}$, Chopitea $\mathrm{A}^{2}$, Sala $\mathrm{P}^{2}$, García del Barrio $\mathrm{MA}^{3}$, Gastaminza $\mathrm{G}^{1}$, Goikoetxea $\mathrm{MJ}^{1}$

${ }^{1}$ Department of Allergy and Clinical Immunology, Clinica Universidad de Navarra, Pamplona, Spain

${ }^{2}$ Department of Oncology, Clínica Universidad de Navarra, Pamplona, Spain

${ }^{3}$ Department of Pharmacy, Clínica Universidad de Navarra, Pamplona, Spain

J Investig Allergol Clin Immunol 2020; Vol. 30(4): 293-295 doi: 10.18176/jiaci.0496

Key words: Desensitization. Omalizumab. Oxaliplatin. Drug allergy.

Palabras clave: Desensibilización. Omalizumab. Oxaliplatino. Alergia a medicamentos.

Omalizumab, an anti-IgE monoclonal antibody, is used as an adjuvant in hymenoptera venom immunotherapy and in induction of oral tolerance in food allergy $[1,2]$. In each of the few cases of desensitization to platins where omalizumab was used, the authors applied a different regimen [3-5]. We present a case of successful oxaliplatin desensitization achieved after a single initial dose of omalizumab in a patient with elevated IgE levels upon reintroduction of oxaliplatin and after severe hypersensitivity reactions (HSRs) during previous desensitization attempts.

A 57-year-old man diagnosed with metastatic sigmoid colon adenocarcinoma was initially treated in 2014 with 9 cycles of folinate, oxaliplatin, and 5-fluorouracil. In 2016, he received 8 cycles of folinate, irinotecan, and 5-fluorouracil. Later that year, his treatment was replaced with gemcitabine, oxaliplatin, and bevacizumab. He tolerated 1 cycle of this regimen. During the second cycle, after receiving gemcitabine and while receiving oxaliplatin, he experienced generalized erythema, cough, dyspnea, and oxygen desaturation. Intradermal testing to oxaliplatin $(0.5 \mathrm{mg} / \mathrm{mL})$ was positive (negative prick test, $5 \mathrm{mg} / \mathrm{mL})$. The results of prick tests $(38 \mathrm{mg} / \mathrm{mL})$ and intradermal tests $(0.38 \mathrm{mg} / \mathrm{mL})$ to gemcitabine were negative. Oxaliplatin was replaced with irinotecan while gemcitabine and bevacizumab were maintained. He tolerated 3 cycles of this new regimen. During the fourth cycle, however, while receiving irinotecan, he presented with anaphylaxis (facial erythema, dyspnea, and pharyngeal pruritus). Intradermal testing with irinotecan $(2 \mathrm{mg} / \mathrm{mL})$ was positive (negative prick test, $20 \mathrm{mg} / \mathrm{mL}$ ).

In 2018, the patient was assigned treatment with folinate, oxaliplatin, 5-fluorouracil, and bevacizumab owing to progression of his tumor. A first 12-step desensitization [6] to oxaliplatin was completed without adverse effects. Premedication with acetylsalicylic acid (ASA) was avoided for this protocol because the patient had a history of angioedema, 
possibly related to this drug (he had tolerated other nonsteroidal antiinflammatory drugs). Oral premedication with montelukast $10 \mathrm{mg}$ was given 2 days before administration and with montelukast $10 \mathrm{mg}$ and ranitidine $300 \mathrm{mg}$ on the day of administration. The oncology department added intravenous premedication with dexamethasone $20 \mathrm{mg}$, dexchlorpheniramine $5 \mathrm{mg}$, and oral acetaminophen $1 \mathrm{~g}$.

In the second desensitization, however, the patient experienced facial erythema, wheezing, dyspnea, oxygen desaturation (89\%), tachycardia (110 bpm), and hypotension $(80 / 50 \mathrm{mmHg})$ during step 10 (7-mg threshold dose). Fortyeight hours later, a new, 16-step attempt failed owing to facial erythema, cough, and oxygen desaturation (89\%) during step 14 (7-mg threshold).

Retrospectively analyzed serum samples from 24 hours before and immediately after the first unsuccessful desensitization showed total IgE levels of 6900 and $7410 \mathrm{kU} / \mathrm{L}$, respectively. Serum tryptase samples were drawn immediately after the 12-step desensitization $(2.03 \mu \mathrm{g} / \mathrm{L})$, and immediately and 2 hours after the 16-step desensitization $(<1.00 \mu \mathrm{g} / \mathrm{L}$ in both cases). Baseline tryptase levels were $1.37 \mu \mathrm{g} / \mathrm{L}$, and eosinophil counts were normal. Skin testing to a standard allergen series and stool parasite tests were negative. He reported no previous history of allergic disease (atopic dermatitis, rhinoconjunctivitis, asthma), alcohol intake, or tuberculosis. A provocation test with $300 \mathrm{mg}$ of ASA was completed without adverse effects

We administered a single, 300-mg dose of subcutaneous omalizumab 19 days before re-attempting desensitization. We followed an 18-step protocol, and $300 \mathrm{mg}$ of ASA was added to the premedication regimen. The patient developed erythema on the face and upper extremities during the 15th step (cumulative oxaliplatin dose, $7.2 \mathrm{mg}$ ), although this responded quickly to intravenous dexchlorpheniramine. Desensitization was restarted 30 minutes later by repeating the previous step and resuming the protocol. There were no new symptoms. This procedure was repeated every 2 weeks for 4 subsequent cycles using $300 \mathrm{mg}$ of omalizumab 2 weeks before each cycle. No new hypersensitivity events occurred, and the tumor stabilized. Variations in total IgE levels are shown in the Figure.

The use of anti-IgE treatment as an adjuvant for desensitization in cases of refractory platin hypersensitivity was first described in 2012 by Cahill et al [3] in a patient with previous failed desensitizations to oxaliplatin and later in 2014 by Ojaimi et al [4] in a case of carboplatin desensitization. Cahill et al used 2 doses of $150 \mathrm{mg}, 1$ every 2 weeks, followed by a 16-step protocol. Ojaimi et al used 3 doses of $300 \mathrm{mg}$, 1 every 2 weeks, prior to desensitization. Prieto-García et al [5] recently used a single $300 \mathrm{mg}$ dose of omalizumab 1 week before desensitization.

To the best of our knowledge, initial total $\operatorname{IgE}$ levels in these reports were not elevated. In our case, however, total IgE levels were consistently high before the first severe HSR, after re-exposure to oxaliplatin. We cannot confirm whether this IgE profile arose because of specific IgE against oxaliplatin or underlying systemic causes, although it defined our patient as a challenging case for desensitization. Regardless, omalizumab was effective as an adjuvant after a single dose, and repeated use resulted in tolerance of all subsequent cycles. This finding

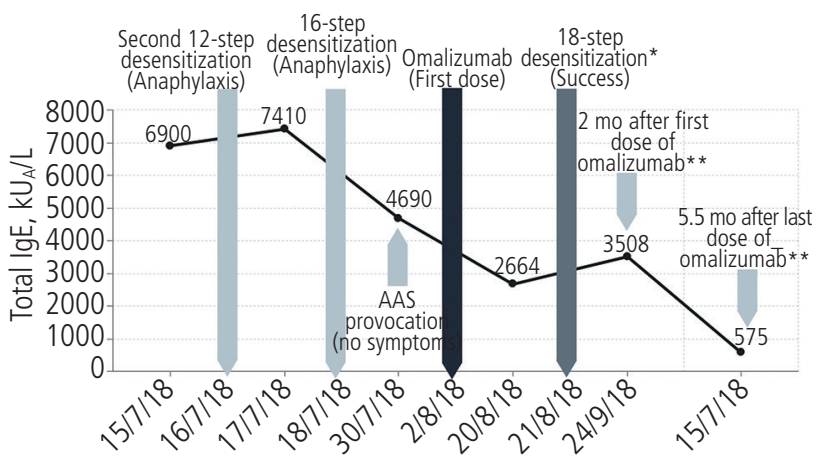

Figure. Patient's progress and variations in IgE levels upon re-exposure to oxaliplatin.

*During this desensitization, $300 \mathrm{mg}$ of acetylsalicylic acid was added to the remainder of the patient's premedication.

**At this point, the patient had received a total of 3 doses of omalizumab (300 mg each). The sample was taken on the day of the third 18-step desensitization.

***This sample was taken 5.5 months after the last dose of omalizumab was administered, and 6 months after the fifth and last 18-step desensitization.

may prove useful when developing omalizumab protocols in such situations.

It is important to mention that, in the case we report, factors other than omalizumab may have led to a favorable outcome. The protocol was extended, and ASA was combined with omalizumab. The use of longer protocols has been described as a means to achieve tolerance to desensitization [7], and this may have played a role in the final outcome. Breslow et al [8] described the efficacy of pretreatment with ASA and montelukast in lessening the severity and the frequency of HSRs to chemotherapy during desensitization. While there is little further evidence proving this protective effect, we cannot rule out its contribution in the present case. Further studies are necessary in patients with a history of failed desensitizations despite modifications to the protocol and complete premedication regimens.

The total IgE profile in the present case, however, leads us to believe that tolerance without anti-IgE treatment would have been difficult, and the variations in total $\operatorname{IgE}$ levels are worth noting (Figure). Prior to receiving anti-IgE treatment, levels had declined from 7410 to $4690 \mathrm{kU} / \mathrm{L}$ in a 2-week period without significant interventions, possibly in part owing to intratest variation [9]. Three weeks after the initial dose of omalizumab, we observed a further decline in total IgE from 4690 to $2664 \mathrm{kU} / \mathrm{L}$, similar to that observed by Ojaimi et al [4]. Finally, total IgE levels had descended to $575 \mathrm{kU} / \mathrm{L}$ at 5 months after the last dose of omalizumab. This clear reduction may partially explain the desensitization to oxaliplatin observed [10].

In cases of advanced cancer where delay in treatment may impair prognosis, time-sparing solutions after unsuccessful desensitizations are clearly beneficial. We report a case of successful desensitization to a platin after a single dose of omalizumab in a patient with a challenging IgE profile. The outcome we report may indicate that the use of single-dose anti-IgE regimens - along with modification of the protocol- 
could be as effective as regimens based on multiple doses before reattempting desensitization. This seems to be true even in patients with elevated total $\operatorname{IgE}$ and a history of repeatedly failed desensitization attempts.

\section{Funding}

The authors declare that no funding was received for the present study.

\section{Conflicts of Interest}

The authors declare that they have no conflicts of interest.

\section{Previous Presentation}

Data from this manuscript were presented at a Poster Discussion Session at the 2019 European Academy of Allergy and Clinical Immunology (EAACI) Congress in Lisbon, Portugal.

Data from this manuscript were presented as an Oral Communication at the 2019 Northern Society of Allergists (AlergoNorte) Congress in Oviedo, Spain.

\section{References}

1. Stretz E, Oppel EM, Räwer HC, Chatelain R, Mastnik S, Przybilla $B$, et al. Overcoming severe adverse reactions to venom immunotherapy using anti-lgE antibodies in combination with a high maintenance dose. Clin Exp Allergy. 2017;47(12):1631-9.

2. Pajno GB, Fernandez-Rivas $M$, Arasi $S$, Roberts $G$, Akdis CA, Alvaro-Lozano $M$, et al; EAACl Allergen Immunotherapy Guidelines Group. EAACl Guidelines on allergen immunotherapy: IgE-mediated food allergy. Allergy. 2018;73(4):799-815.

3. Cahill KN, Harrison $P$, de Asis $M$, Castells $M$. Use of Omalizumab to Achieve Successful Desensitization after Oxaliplatin Anaphylaxis [Abstract]. J Allergy Clin Immunol. 2012;129(2) Supplement:AB103.

4. Ojaimi S, Harnett PR, Fulcher DA. Successful carboplatin desensitization by using omalizumab and paradoxical diminution of total IgE levels. J Allergy Clin Immunol Pract. 2014;2(1):105-6.

5. Prieto-García A, Noguerado B, Rojas P, Torrado I, RodríguezFernández A, Tornero P. Unexpected Anaphylaxis After Completing a Desensitization Protocol to Oxaliplatin: Successful Adjuvant Use of Omalizumab. J Investig Allergol Clin Immunol. 2019;29(1):53-5.

6. Castells MC, Tennant NM, Sloane DE, Hsu Fl, Barrett NA, Hong DI, et al. Hypersensitivity reactions to chemotherapy: outcomes and safety of rapid desensitization in 413 cases. J Allergy Clin Immunol. 2008;122(3):574-80.

7. Liu A, Fanning L, Chong H, Fernandez J, Sloane D, Sancho-Serra $M$, et al. Desensitization regimens for drug allergy: state of the art in the 21st century. Clin Exp Allergy. 2011;41(12):167989.

8. Breslow RG, Caiado J, Castells MC. Acetylsalicylic acid and montelukast block mast cell mediator-related symptoms during rapid desensitization. Ann Allergy Asthma Immunol. 2009;102(2):155-60.

9. Hamilton RG, Oppenheimer J. Serological IgE Analyses in the Diagnostic Algorithm for Allergic Disease. J Allergy Clin Immunol Pract. 2015;3(6):833-40.

10. Lowe PJ, Tannenbaum $S$, Gautier $A$, Jimenez $P$. Relationship between omalizumab pharmacokinetics, IgE pharmacodynamics and symptoms in patients with severe persistent allergic (IgE-mediated) asthma. Br J Clin Pharmacol. 2009;68(1):61-76.

Manuscript received October 20, 2019; accepted for publication February 14, 2020.

Paola Quan

Clínica Universidad de Navarra Avenida de Pío XII, 36 31008 Pamplona, Navarra Spain

E-mail: pquan@unav.es 